Check for updates

Cite this: RSC Adv., 2019, 9, 28222

Received 13th July 2019

Accepted 2nd September 2019

DOI: 10.1039/c9ra05359j

rsc.li/rsc-advances

\section{Designing dendronic-Raman markers for sensitive detection using surface-enhanced Raman spectroscopy $\dagger$}

\author{
Priyanka Jain, ${ }^{\text {ab }}$ Robi Sankar Patra, ${ }^{c}$ Sridhar Rajaram ${ }^{\star b d}$ \\ and Chandrabhas Narayana (iD *ab
}

Surface-Enhanced Raman Spectroscopy (SERS) is well-established as a tool for bio-diagnostics but is often limited by analyte sensitivity and the need for specialized substrates. Signal enhancement can be achieved by attaching multiple Raman markers to a single analyte. Dendronic frameworks with multiple Raman markers attached to the periphery offer an opportunity to examine this idea. In this article, dendrons with thiophenol groups on their periphery were synthesized and tested as a SERS analyte. For this study, simple gold nanoparticles ( $60 \mathrm{~nm}$ ) were used as a substrate. A $10^{2}$ fold enhancement in detection was observed upon going from a mono-thiophenol (MT) to a tetra-thiophenol (TT). Dendronic Raman markers increased the probability of SERS occurrence at lower concentrations when compared to a single Raman active molecule. This strategy extends the applicability of SERS, as these analyte molecules can be just mixed or drop-casted on any kind of SERS substrate.

\section{Introduction}

Sensitive detection for biological applications, especially diagnostics, has been a widespread area of interest. Most disease states commence with slight changes in the cellular processes which magnifies with disease progression. ${ }^{1}$ Thus, early diagnosis and regular monitoring becomes paramount for enhancing treatment efficacy. In view of this, there have been numerous reports exploiting SERS for disease diagnostics. ${ }^{2-5}$ SERS has been extensively used for its ability to obtain a molecular signature and achieve trace detection of analytes. ${ }^{6-9}$ With the possibility of customized miniaturized Raman spectrometers, ${ }^{\mathbf{1 0 , 1 1}}$ surface-enhanced Raman spectroscopy becomes a very important tool for bio-diagnostics.

For early diagnosis, where the biological analyte concentration is low, detecting a Raman reporter labelled to a biomolecule becomes advantageous. Various strategies have been applied to functionalize a Raman reporter to a specific linker that can target specific biomolecules. ${ }^{12-14}$ Cao et al. performed a multiplexed detection of oligonucleotide targets using gold

${ }^{a}$ Chemistry and Physics of Materials Unit, Jawaharlal Nehru Centre for Advanced Scientific Research (JNCASR), Bengaluru, India.E-mail: cbhas@jncasr.ac.in ${ }^{b}$ School of Advanced Materials, JNCASR, Bengaluru, India.E-mail: rajaram@jncasr.ac. in

${ }^{c}$ New Chemistry Unit, JNCASR, Bengaluru, India

${ }^{d}$ International Centre for Materials Science, JNCASR, Bengaluru, India

$\dagger$ Electronic supplementary information (ESI) available: Synthesis procedures and spectroscopic data, SERS experimental method, TEM image and extinction spectra of Au nanoparticles and table for SERS intensities of $1004 \mathrm{~cm}^{-1}\left(v_{12}\right)$ mode for synthesized thiophenol derivatives. See DOI: $10.1039 / \mathrm{c} 9 \mathrm{ra05359j}$
(Au) nanoparticles labelled with oligonucleotides and dye molecules. ${ }^{2}$ Later, Ranga et al. developed a high sensitivity assay for HIV-1 subtype detection using Surface-Enhanced Resonant Raman Spectroscopy (SERRS). ${ }^{15}$ These assays were highly specific and manifested high sensitivity up to femtomolar level. However, early diagnosis of disease progression requires this limit to be pushed further.

The overall sensitivity of these strategies is influenced by the signal obtained from the Raman marker. SERS signal intensity has mainly been enhanced by substrate engineering to maximize interaction of Raman reporters with the surface of the

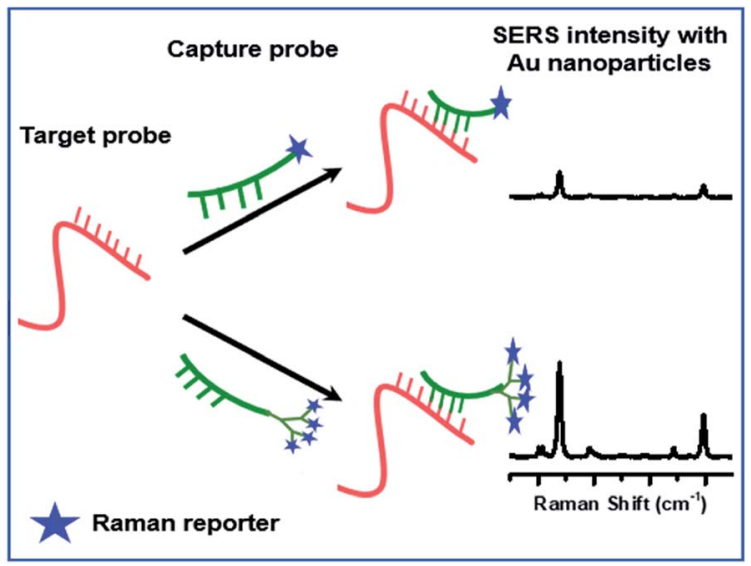

Fig. 1 Proposed hypothesis for the SERS based oligonucleotide assay. 
<smiles>CC(C(=O)OCc1ccccc1)(C(=O)OCc1ccc(S)cc1)C(=O)OC(=O)Cc1ccc(S)cc1</smiles>

Monothiophenol (MT) Dithiophenol (DT)<smiles>CCOOCCOC(=O)Cc1ccc(S)cc1</smiles>

Fig. 2 Structures of thiophenol derivatives synthesized.

substrate. ${ }^{\mathbf{1 6 - 1 8}}$ However, the processes involved in engineering the substrate are cumbersome and may not be scalable. To achieve ultra-sensitive SERS detection, other techniques including analyte modification along with hotspot engineering is important. ${ }^{19}$ In our quest to detect low levels of viral RNA
(Target probe), we hypothesized that a stronger signal could be obtained when multiple Raman markers are attached to a detector strand (Capture probe) as opposed to a single Raman marker as shown in Fig. 1. Before this can be reduced to practice, the level of enhancement that can be obtained by attaching multiple Raman markers to an analyte has to be determined. To probe this, we chose to determine the detection limit of Raman markers attached to the periphery of a dendron.

Dendrons are macromolecules which are highly branched and monodisperse in nature. A large number of functional groups can be introduced in the periphery of the dendrons with precise structural control. ${ }^{20-22}$ We chose the 2,2-bis(methylol)propionic acid (Bis-MPA) dendron as a scaffold for connecting the oligo and the Raman marker. The oligo can be potentially linked to the focal point of the dendron, while signal enhancement can be achieved by attaching multiple copies of the Raman marker in the periphery. As a proof of principle, we have synthesized a Bis-MPA dendron with a benzyl group in the focal point and thiophenol as the Raman reporter on the periphery. This would clarify the potential for signal enhancement that is possible with multiple Raman markers. Other Raman markers will be explored in future. The analyte with tetra-thiophenol substitution was detectable consistently up to a $10^{-7} \mathrm{M}$ concentration, which was a 100 -fold enhancement of detection concentration when compared to a mono thiophenol. In addition, these results were obtained using simple colloidal $\mathrm{Au}$ nanoparticles as SERS substrate. The nanoparticles were prepared using kinetic seeded-growth method. ${ }^{23}$

\section{Results and discussions}

Synthesis of Bis-MPA dendrons were carried out as reported in the literature. $^{24-27}$ Thiophenol (TP) was chosen as the Ramanactive counterpart in the dendronic structure owing to the high affinity of thiol molecules towards Au. ${ }^{28}$ Even though it is a relatively poor scatterer when compared with dyes like rhodamine $6 \mathrm{G}$, we chose it for the ease-of-synthesis. The<smiles>CC(C)C(=O)OC(=O)C(CO)(CO)C(=O)OC(=O)C(CO)(CO)C(=O)OC(=O)C(CO)(CO)C(=O)OCc1ccc(S)cc1</smiles>

Tetrathiophenol (TT)

Scheme 1 Synthesis of thiophenol derivatives. 
structures of the TP derivatives are shown in Fig. 2. Coupling of 4-S-trityl mercapto phenyl acetic acid with benzyl alcohol using DCC ( $N, N^{\prime}$-dicyclohexylcarbodiimide) yielded the trityl protected mono-thiophenol derivative (Scheme 1). Similarly, coupling with the Bis-MPA derived diol and the G1 dendron gave the protected di- and tetra-thiophenol derivatives, respectively. The three derivatives were de-protected using trifluoroacetic acid (TFA) and triisopropylsilane (TIPS) to yield the corresponding thiophenol derivatives (Scheme 1). Details of synthesis and characterization of the Bis-MPA dendronic Raman markers are reported in the ESI. $\dagger$

Fig. 3(a) shows the SERS spectra of benzyl 4-mercapto phenylacetic acid (MT) at various concentrations. The MT system was detectable up to $10^{-5} \mathrm{M}$ concentration as expected and seen in previous reports. ${ }^{22,29}$ The peaks at 1006, 1018, 1080, and $1595 \mathrm{~cm}^{-1}$ are characteristic peaks of TP molecule and assigned to in-plane ring deformation, in-plane ring deformation and C-C symmetric stretch, C-C asymmetric stretch and C-S stretch, and $\mathrm{C}-\mathrm{C}$ symmetric bending modes respectively. ${ }^{30,31}$ For comparing with the other systems chosen for this study, we normalized all spectra with respect to $1078 \mathrm{~cm}^{-1}$ peak. All spectra shown in Fig. 3 are background corrected with respect to the $\mathrm{Au}$ nanoparticle (see $\mathrm{ESI} \dagger$ for synthesis procedure) and solvents used. It is to be noted that the origin of the peak around $1540 \mathrm{~cm}^{-1}$ (marked by * in Fig. 3(a)) is unclear and suspected to come from the ethanol and citrate ions interaction with Au nanoparticles.

The SERS studies were then extended to di-thiophenol (DT) and tetra-thiophenol (TT) derivatives, shown in Fig. 3(b) and (c) respectively. In the case of DT, only a nominal increase in signal enhancement was observed. On the other hand, TT displays a steep enhancement of the detection limit and is detectable up to a concentration of $10^{-7} \mathrm{M}$ concentration. A $10^{2}$-fold increase in detection limit was recorded consistently. It is important to understand these results in order to develop better strategies based on them.

Although a comprehensive theory of SERS has not been developed yet, it is now well accepted that signal enhancement in SERS occurs due to electromagnetic enhancement and chemical enhancement. ${ }^{32,33}$ Further increase in signal intensity can be obtained by formation of hotspots. ${ }^{34}$ In this scenario, it is unlikely that all Raman markers contribute to the SERS signal, which makes hotspots generation quite significant especially in low concentration regime. Tailoring the substrate provides more opportunity for such environment in a tiny region and is advantageous when having static detection of analytes. ${ }^{35,36}$ However, in the case of detection under flow-conditions, this will be difficult to achieve without intricate SERS structures in the flow chambers. In our study, the molecules (MT, DT and TT) are incubated with the Au nanoparticle solution in the ratio of $1: 100$ by volume for one hour. Post-incubation, these colloidal solutions were centrifuged to form a pellet (residue from centrifugation) generating hotspots for the nanoparticles regardless of the analyte molecules. The tiny microdroplet (or pellet) is then irradiated with a laser source of about 1-2 $\mu \mathrm{m}$ spot size thus, probing an average number of hotspots. Although these hotspots have a non-uniform distribution, ${ }^{37}$ we obtain a similar reproducible average. It is to be noted that, not all hotspots generated are occupied by the analyte molecules considering the ratio of the analyte to nanoparticle. Such colloidal nanoparticles also lead to random orientations of the analyte molecules on their surface and these hotspots. ${ }^{38}$ This is
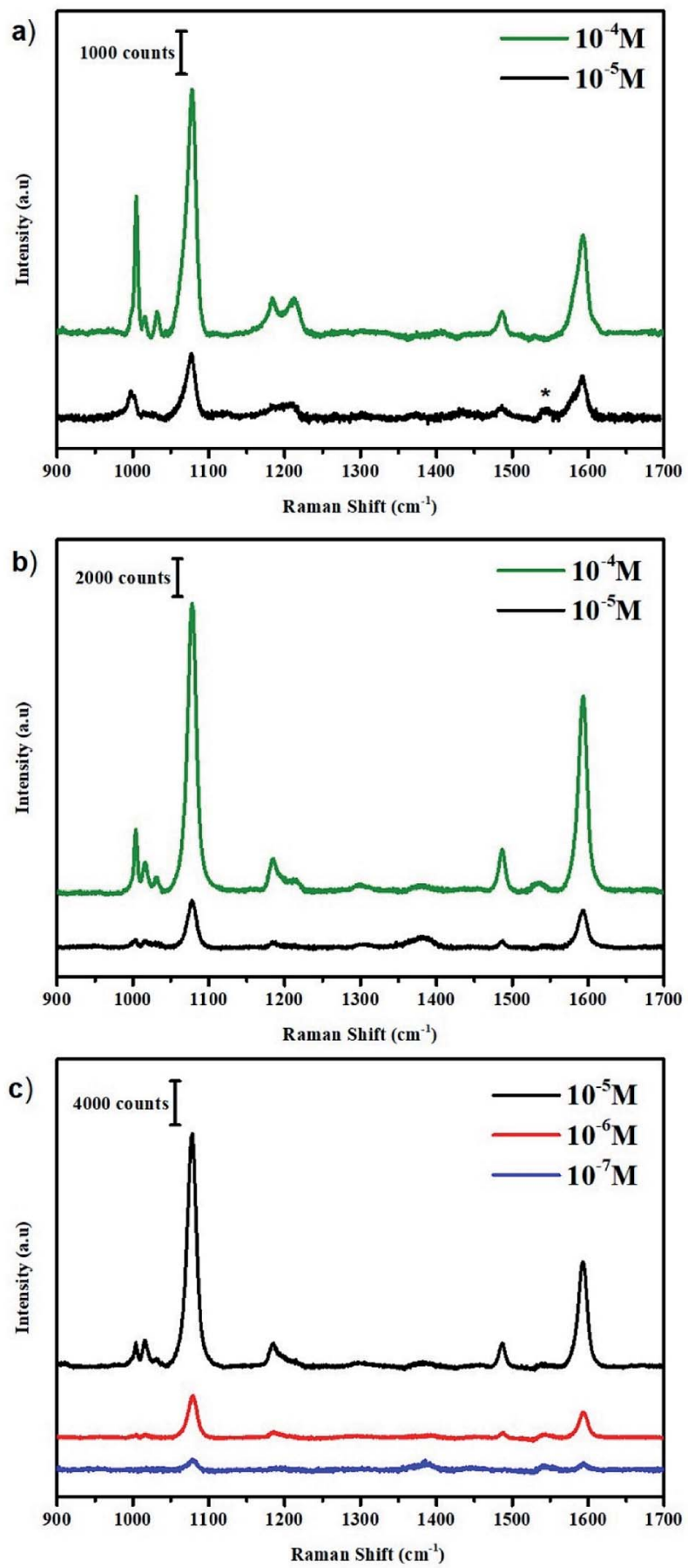

Fig. 3 (a) SERS spectrum of benzyl-4-mercapto phenylacetic acid (MT) solution at different concentrations. (b) SERS spectrum of dithiophenol (DT) derivative solution at different concentrations. (c) SERS spectrum of tetra-thiophenol (TT) derivative solution at different concentrations. The laser wavelength used was $633 \mathrm{~nm}$ with $3 \mathrm{~mW}$ laser power at the sample and $10 \mathrm{~s}$ acquisition time. 
where increasing the number of Raman markers per detector probe increases the chance of each probe getting detected. ${ }^{39}$ In this backdrop we will explain our results.

The molecules in question will have similar fundamental interaction with the nanoparticles (Au-thiol) but will vary in the number of analytes per hotspot. By confining large number of thiophenol molecules into a dendronic framework, we increase the possibility of hotspot occupancy as seen in the top view of Fig. 4. Labels 1, 2 and 4 mark the possible number of active sites interacting with the Au nanoparticle for TT derivatives under hotspot conditions. It is to be noted that, all the possibilities will not exist simultaneously under same hotspot conditions because compared to the neighbouring metallic surface, the surface area of a hotspot is very small. ${ }^{37}$ Hence, Fig. 4 depicts the various random possibilities of the TT derivative occupying hotspot (depending on the TP ring orientation which is discussed subsequently). In the case of MT and DT derivatives the odds of occupying hotspot are lesser compared to the TT derivative because the chances of unbound TPs for TT derivative are higher compared to the former derivatives. These unbound TPs can bind to other nanoparticles in the hotspots once formed due to pelletisation. This is the reason, we do not observe a large increase in SERS signal of the DT derivative whereas, it increases drastically for the TT derivative. A 100-fold enhancement in the detection limit of the analyte molecule supports our argument that not all Raman markers contribute to the SERS signal equally. These results emphasize that the possibility of having Raman markers in the right environment increases non-linearly with increase in the number of Raman markers confined per analyte giving a large SERS enhancement. While both the number and orientation of the analyte which are proximal to the nanoparticles and its hotspots affect the SERS signal, in the low concentration regime (where only a few analytes are present) further constraining these TP molecules into a dendronic system drastically influences this number orienting in the right position.

In order to obtain an understanding of the orientations of the three systems under SERS conditions, we look through the well-known fundamental modes associated with TP. Carron et al. determined the orientation of the benzene ring by looking at the ring vibrations classified as $\mathrm{a}_{1}, \mathrm{a}_{2}, \mathrm{~b}_{1} \& \mathrm{~b}_{2}$, assuming a $C_{2 \mathrm{v}}$ symmetry. ${ }^{40}$ The SERS spectra contain mostly peaks of the $a_{1}$ modes at 1003, 1078, 1184, 1485 and $1593 \mathrm{~cm}^{-1}$ whereas; the $\mathrm{a}_{2}$ and $b_{1}$ modes obtained were very weak making them unsuitable for analysis. The $a_{1}$ modes include a linear combination of polarizability tensors $\alpha_{x x}, \alpha_{y y}, \alpha_{z z}$, hence making it difficult to include for analysis to determine orientation. However, the $a_{1}$ mode associated with only the phenyl ring breathing could be used for determining the orientation. ${ }^{40}$ According to surface selection rule, the in-plane bending modes would be quite strong for a perpendicular orientation with respect to the nanoparticle surface and vice versa ${ }^{41} \mathrm{Li}$ et al. concluded that the in-plane modes at $1021\left(v_{18 \mathrm{a}}\right)$ and $988 \mathrm{~cm}^{-1}\left(v_{12}\right)$ were strongly related to the orientation of the adsorbed 4-mercaptophenyl boronic acid (MPBA) molecule and consider them to be orientation-sensitive marker bands. ${ }^{42}$

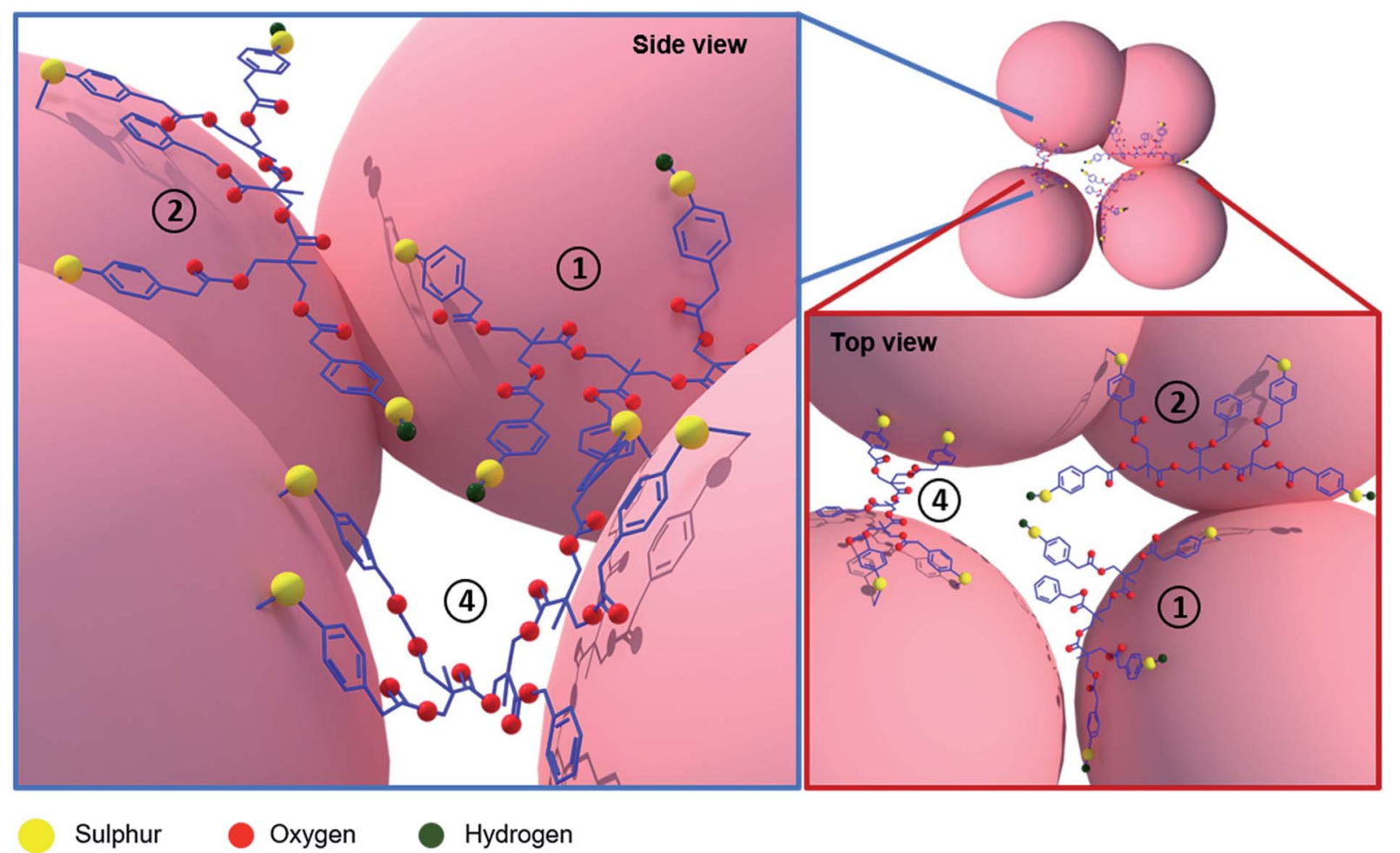

Fig. 4 Possible orientations and interaction of $\Pi$ derivative with the nanoparticles. Labels 1, 2 and 4 depict the number of active sites which may bind to the nanoparticle under different hotspot conditions for the $T T$ derivative. 
With this knowledge, we closely look at the $1006 \mathrm{~cm}^{-1}$ band (normalized w.r.t $1078 \mathrm{~cm}^{-1}$ ) corresponding to the $v_{12}$ mode in case of MT which downshifts to $1004 \mathrm{~cm}^{-1}$ in case of DT and TT. From Table $\mathrm{S} 1, \uparrow$ we clearly observe the decrease in the intensity of $v_{12}$ mode with increasing number of TP molecules. For TT derivative, the signal intensity of the $1004 \mathrm{~cm}^{-1}$ is 0.1 indicating a nearly flat orientation on the surface of the Au nanoparticle. At $10^{-5} \mathrm{M}$ concentration, a near flatter orientation of the TT derivative compounded with multiple Raman markers indicates a lower coverage per unit area of Au nanoparticle as compared to when in a perpendicular orientation. However, we observe a much-enhanced SERS signal at the same point which indicates more number of molecules sitting in hotspot conditions because of the dendronic geometry as shown in the side view of Fig. 4. In this concentration regime, a MT system (where the active molecules are all independent, less in number and not constricted) will have a lesser probability of occupying such hotspots as they would tend to spread out resulting in a poor signal being observed. Dendronic geometry not only increases the probability of obtaining a SERS signal thus, pushing the detection limit by 100 -fold but also allows the molecules to occupy more hotspots sites due to their constrained framework.

The scheme envisaged in this letter to demonstrate SERS signal enhancement for trace detection by using multiple Raman markers, can be performed on any SERS substrates or nanoparticles and can be adapted in both static and dynamic systems. Here, the experiment was carried just by mixing the two solutions, centrifuging and dropping on a glass slide with no prior sample preparation. The obtained spectra are a result of these "non-ideal" molecules and conditions used, hence making the strategy extremely promising. It also shows that this is a better solution than just tailoring the SERS substrates to provide better hotspot conditions. These results can be drastically improved by using much better SERS markers thereby, lowering the detection limit (femto to atto molar concentrations). Since, it is a chemical strategy, the analyte can always be modified to better scattering dye molecules like Rhodamine 6G. Such dye molecules would require a more sophisticated purification technique than normal column chromatography; they inherently give better SERS enhancement for a single molecule itself.

\section{Conclusions}

We have developed an analyte strategy for improved detection of biomarkers. The model system was synthesized using a simple coupling reaction carried between the Bis-MPA dendrons and TP molecules yielded the di-thiophenol and tetrathiophenol Raman markers. From the SERS studies a $10^{2}$-fold enhancement in the detection limit was observed as we go from a MT system to a TT system using simple Au colloidal nanoparticles. It is proposed that, by bringing the analyte molecule into a dendronic framework, we increase the probability of at least one of the four Raman markers to achieve a signal and pushing the detectable concentration by 100 times. The applicability of the strategy is widened by ease of investigation on any kind of SERS substrate in future. Alternatively, any type of biomarkers like rhodamine $6 \mathrm{G}$ can be linked to the dendrons for further enhancement. A facile synthesis with an oligonucleotide can be carried on these molecules by de-protecting the other end of the dendronic system, thus making them a superior biomarker strategy in the future.

\section{Conflicts of interest}

There are no conflicts to declare.

\section{Acknowledgements}

Authors S. R. and C. N. would like to acknowledge Technical Research Centre (TRC) of JNCASR funded by Department of Science and Technology (DST). Authors P. J. and R. S. P. would like to acknowledge Council of Scientific and Industrial Research (CSIR) for fellowship.

\section{Notes and references}

1 T. J. Moore, A. S. Moody, T. D. Payne, G. M. Sarabia, A. R. Daniel and B. Sharma, Biosensors, 2018, 8, 46.

2 Y. C. Cao, R. Jin and C. A. Mirkin, Science, 2002, 297, 15361541.

3 S. S. R. Dasary, A. K. Singh, D. Senapati, H. Yu and P. C. Ray, J. Am. Chem. Soc., 2009, 131, 13806-13812.

4 M. Kahraman, E. R. Mullen, A. Korkmaz and S. WachsmannHogiu, Nanophotonics, 2017, 6, 831-852.

5 L.-J. Xu, Z.-C. Lei, J. Li, C. Zong, C. J. Yang and B. Ren, J. Am. Chem. Soc., 2015, 137, 5149-5154.

6 E. J. Blackie, E. C. Le Ru and P. G. Etchegoin, J. Am. Chem. Soc., 2009, 131, 14466-14472.

7 M. Fleischmann, P. J. Hendra and A. J. McQuillan, Chem. Phys. Lett., 1974, 26, 163-166.

8 K. Kneipp, H. Kneipp and J. Kneipp, Acc. Chem. Res., 2006, 39, 443-450.

9 S. Nie and S. R. Emory, Science, 1997, 275, 1102-1106.

10 G. V. P. Kumar and C. Narayana, US Pat., US 2009/0244534, 2009.

11 G. V. P. Kumar and C. Narayana, Curr. Sci., 2007, 93, 778781.

12 K. Faulds, R. Jarvis, W. E. Smith, D. Graham and R. Goodacre, Analyst, 2008, 133, 1505-1512.

13 J. M. Li, C. Wei, W. F. Ma, Q. An, J. Guo, J. Hu and C. C. Wang, J. Mater. Chem., 2012, 22, 12100-12106.

14 L. Sun, C. Yu and J. Irudayaraj, Anal. Chem., 2007, 79, 39813988.

15 U. K. Ranga, C. Narayana and J. Narayana, US Pat., US 2010/ 0028858, 2010, 1.

16 M. P. Cecchini, V. A. Turek, J. Paget, A. A. Kornyshev and J. B. Edel, Nat. Mater., 2012, 12, 165-171.

17 P. A. Mosier-boss, Nanomaterials, 2017, 7, 142.

18 S. Shanmukh, L. Jones, J. Driskell, Y. Zhao, R. Dluhy and R. A. Tripp, Nano Lett., 2006, 6, 2630-2636.

19 H. K. Lee, Y. H. Lee, C. S. L. Koh, G. C. Phan-Quang, X. Han, C. L. Lay, H. Y. F. Sim, Y. C. Kao, Q. An and X. Y. Ling, Chem. Soc. Rev., 2019, 48, 731-756. 
20 P. Antoni, D. Nyström, C. J. Hawker, A. Hult and M. Malkoch, Chem. Commun., 2007, 2249-2251, DOI: 10.1039/B703547K.

21 A. Carlmark, C. Hawker, M. Malkoch and C. Hawker, Chem. Soc. Rev., 2009, 38, 352-362.

22 M. V. Walter, M. Malkoch, M. V. Walter and M. Malkoch, Chem. Soc. Rev., 2012, 41.

23 N. G. Bast, J. Comenge and V. Puntes, Langmuir, 2011, 27, 11098-11105.

24 S. Castelar, J. Barberá, M. Marcos, P. Romero, J.-L. Serrano, A. Golemme and R. Termine, J. Mater. Chem. C, 2013, 1, 7321-7332.

25 N. Feliu, M. V. Walter, M. I. Montañez, A. Kunzmann, A. Hult, A. Nyström, M. Malkoch and B. Fadeel, Biomaterials, 2012, 33, 1970-1981.

26 T. Jiang, R. Liu, X. Huang, H. Feng, W. Teo and B. Xing, Chem. Commun., 2009, 1972-1974, DOI: 10.1039/b818853j.

27 A. S. Sayyad and P. M. Ajayan, Nanoscale, 2011, 3605-3608, DOI: $10.1039 / \mathrm{c} 1 \mathrm{nr} 10579 \mathrm{e}$.

28 F. Sun, D. D. Galvan, P. Jain and Q. Yu, Chem. Commun., 2017, 53, 4550-4561.

29 G. Kumari and C. Narayana, J. Phys. Chem. Lett., 2012, 3, 1130-1135.

30 S. Li, D. Wu, X. Xu and R. Gu, J. Raman Spectrosc., 2007, 38, 1436-1443.

31 M. Osawa, N. Matsuda, K. Yoshii and I. Uchida, J. Phys. Chem., 1994, 98, 12702-12707.
32 E. C. Le Ru and P. G. Etchegoin, Principles of surface-enhanced Raman spectroscopy and related plasmonic effects, Elsevier, Amsterdam, 2009, pp. 299-365, DOI: 10.1016/B978-0-44452779-0.00012-X.

33 B. N. J. Persson, K. Zhao and Z. Zhang, Phys. Rev. Lett., 2006, 96, 207401.

34 X. Wang, M. Li, L. Meng, K. Lin, J. Feng, T. Huang, Z. Yang and B. Ren, ACS Nano, 2014, 8, 528-536.

35 Q. Yu, S. Braswell, B. Christin, J. Xu, P. M. Wallace, H. Gong and D. Kaminsky, Nanotechnology, 2010, 21, 355301.

36 Q. Yu, P. Guan, D. Qin, G. Golden and P. M. Wallace, Nano Lett., 2008, 8, 1923-1928.

37 E. C. Le Ru, E. Blackie, M. Meyer and P. G. Etchegoint, J. Phys. Chem. C, 2007, 111, 13794-13803.

38 W. Xu, X. Ling, J. Xiao, M. S. Dresselhaus, J. Kong, H. Xu, Z. Liu and J. Zhang, Proc. Natl. Acad. Sci. U. S. A., 2012, 109, 9281-9286.

39 The compaction for the new analytes with nanoparticles can be achieved by techniques like applying magnetic field for magnetic core gold shell nanoparticles.

40 K. T. Carron and L. G. Hurley, J. Phys. Chem., 1991, 95, 99799984.

41 M. Moskovits, J. Chem. Phys., 1982, 77, 4408-4416.

42 S. Li, Q. Zhou, W. Chu, W. Zhao and J. Zheng, Phys. Chem. Chem. Phys., 2015, 17, 17638-17645. 